\title{
Electron irradiation damage to aromatic compounds
}

\author{
P. Li and R.F. Egerton
}

\section{Physics Department, University of Alberta, Edmonton, Canada T6G 2J1}

Radiation damage is of continuing interest to electron microscopists, most recently in connection with aberration-corrected TEM's and low-voltage scanning microscopes. It has been suggested that damage to $\pi$-bonded organic compounds requires carbon K-shell excitation, based on similarity of the cross sections for damage and for $\mathrm{K}$-shell ionization [1] or on the rate of fading of the diffraction pattern as a function of incident-electron energy $\mathrm{E}_{0}[2,3]$. If so, a low-voltage SEM could examine such materials without damage, while at higher $\mathrm{E}_{0}$ there would be negligible effect from low-energy secondary electrons, leading to high spatial resolution if these materials were used as electron-beam resists.

We have investigated the radiation damage in coronene, rubrene and other aromatic compounds according to several criteria. Loss of long-range order (crystallinity) was monitored from fading of electron-diffraction patterns. Disruption of the aromatic ring within each molecule was measured from decay of the $6 \mathrm{eV}$ energy-loss peak, representing $\pi-\pi^{*}$ transitions. These measurements were performed a TEM equipped with a PEELS system and CCD camera. Radiation sensitivity can be represented by a damage cross section $\sigma=\mathrm{e} / \mathrm{D}_{\mathrm{c}}$ where e is the electronic charge and $\mathrm{D}_{\mathrm{c}}$ is a characteristic dose for diffraction or $\pi$-peak fading. Results for $\mathrm{E}_{0}=200 \mathrm{keV}$ are shown in Fig. 1. Loss of crystallinity is seen to be less rapid when the irradiation takes place at lower temperature [4]. As expected for aromatic molecules [1], though not for $\mathrm{C}_{60}$ [5], loss of the $\pi$-resonance peak has a lower cross section, i.e. a higher dose than needed for loss of crystallinity.

Decay of the cathodoluminescence (CL) signal was measured using a Philips 505 SEM operated with accelerating voltages between $1 \mathrm{kV}$ and $30 \mathrm{kV}$. Landing energies below $1000 \mathrm{eV}$ were obtained by applying negative voltage to the specimen stage. Emitted light was collected by a fibre-optic spectrometer system or by a photomultiplier tube. Cross sections for CL decay are shown as solid circles in Fig. 1. The dashed curve represents the cross section per molecule for all inelastic scattering; its approximate match with the CL data suggests that the molecular energy-level structure can be disrupted (leading to loss of light emission) by a single inelastic event occurring within each molecule. The fact that $\mathrm{CL}$ cross sections (for $\mathrm{E}_{0}>1 \mathrm{keV}$ ) are higher than the total-inelastic cross section may be due to plural scattering and the fact that some inelastic collisions generate several secondary electrons, which then damage adjacent molecules [6]. The decrease in CL cross section for $\mathrm{E}_{0}<1 \mathrm{keV}$ reflects the lower energy (per primary electron) available for bond breakage and the production of secondaries.

Open circles in Fig. 1 show cross sections for loss of crystallinity measured for coronene by the Cavendish group [2,3]. They match (in absolute value) the calculated cross section for one K-shell ionization per molecule (solid curve in Fig. 1), suggesting that a single K-ionization per molecule is sufficient to cause randomization of the intermolecular spacing and orientation (loss of crystallinity). This argument is supported [2,3] by the sharp reduction in cross section which was observed as the incident energy was reduced to values approaching the K-shell ionization energy $(\sim 285 \mathrm{eV})$. However, care must be exercised in interpreting measurements in which crystallinity is determined subsequent to irradiation, using electrons of higher energy. For $\mathrm{E}_{0}<500 \mathrm{eV}$, the electron range becomes less than the specimen thickness, so electron irradiation can be expected to leave an undamaged layer which is subsequently detected by transmission diffraction and could be mistaken for lack of damage. This 
possibility occurs at higher $\mathrm{E}_{0}$ (for a given average thickness) if the specimen has a porous granular structure. To produce specimens with constant thickness, we vacuum-deposited the organic materials onto a cooled $(100 \mathrm{~K})$ substrate. However, this procedure results in amorphous films which cannot be used for diffraction measurements.

[1] M.S. Isaacson, in Physical Aspects of Electron Microscopy and Microbeam Analysis, Wiley 1975. [2] A. Howie et al., Inst. Phys. Conf. Ser. No. 90 (EMAG 87), 155.

[3] M.N. Mohd Muhid et al., Proc. IVth Asia-Pacific Conference and Workshop on Electron Microscopy, Bangkok, 1988, 327.

[4] J.R. Fryer et al., Ultramicroscopy 40 (1992) 163-169.

[5] R.F. Egerton and M. Takeuchi, Applied Physics Letters 75 (1999) 1884.

[6] K. Siangchaew and M. Libera, Philosophical Magazine 80 (2000) 1001.

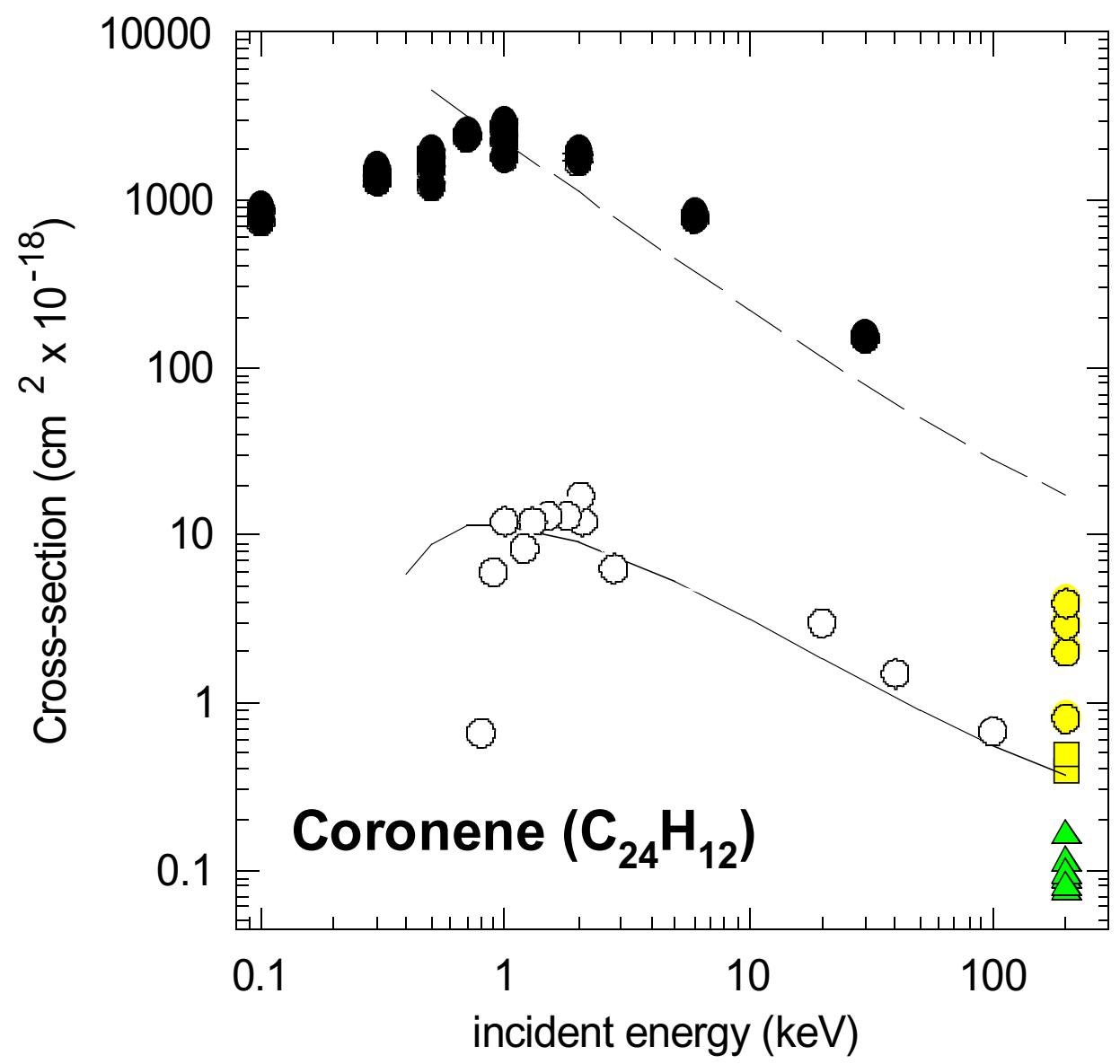

FIG. 1. Cross sections for cathodoluminescence decay (black circles), loss of crystallinity (open circles and squares) and decay of the $6 \mathrm{eV}$ energy-loss peak (triangles), compared with calculated cross sections for a single K-shell (solid curve) or valence-electron (dashed curve) excitation per molecule. 